\title{
Promoting global collaboration for brain health research
}

\author{
Liping Liu and colleagues discuss the challenges of global collaboration for brain health research \\ and promising future opportunities for improvement of brain health worldwide
}

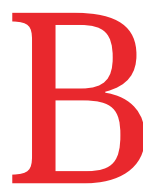
rain science is still in a discovery phase because of our limited knowledge of basic nervous system structure and function. We need a broader perspective of delivering meaningful outcomes to patients with neurological disorders and greater understanding of the mechanisms that underlie development of neural circuitry, how neurons encode and retrieve information, and how information interacts from one neuron to another. Knowledge of how brain activity gives rise to complex behaviours and how it adapts to external and internal changes is limited. Superficial understanding of the various senses, emotions, and cognitive functions-thinking, choice, and even consciousness-promise innovative solutions in areas such as health, education, and 21st century economics. ${ }^{1}$ With the increasing burden of major brain diseases across the world, we need to find the most effective means to comprehensively apply modern biotechnology and to solve problems in clinical medicine.

Neuroscience is entering a new era of collaboration, in which successful new technologies, generated by large scientific projects across the world, will have a dramatic impact not only on medical science but on economics and society as well. In 2013, the US government launched the Brain Research Through Advancing

\section{REY MESSAGES}

- Limited research resources and knowledge of nervous system structure and function make it difficult to achieve a better outcome for those with neurological disorders

- A new collaborative era of neuroscience encourges broad distribution of novel technologies and opens accessibility of the data generated worldwide

- Further priorities and strategies to promote global collaboration on key brain health and initiatives are warranted to increase the opportunities
Innovative Neurotechnologies (BRAIN) initiative and put forward a national brain science project. This initiative complemented the Human Brain Project in the European Union and was shortly joined by the Brain/MINDS programme in Japan and several other national initiatives from Korea, China, Canada, Australia, and the International Brain Research Organization. Coordinating these successful international programmes and encouraging broad distribution of new technologies and open accessibility of the data generated will increase their value, while promoting creativity and expertise from every source. Multidisciplinary science that leverages translational research is critical to the success of these endeavours, along with the establishment of distribution systems and sharing mechanisms of technology on human data. In support of these goals the International Brain Initiative was formed to coordinate global BRAIN projects. ${ }^{2}$. This may facilitate comparability of the data and reduce economic and social disease burden. The complexity of the human brain is reflected in how its molecules, cells, circuits, and systems enable humans to perceive, recognise, and communicate with each other, as well as to understand how our brain confers our individual identity and enables us to contemplate our place in the natural world. ${ }^{3}$ The ambitious goal of understanding the brain is being approached in various projects. Paramount to this process of tackling all the objectives is the commitment to collaboration between government and non-government organisations and integration of basic and clinical translational brain research. ${ }^{4}$

\section{Clinical promise}

Global brain initiatives to map, monitor, and modulate brain activity will lead to a host of clinical applications. Our colleagues from the stroke and neurocritical care community look forward to technologies that can improve ability to diagnose and successfully intervene to prevent and treat severe brain injury as well as enhance the brain's capability for rewiring for improved function. The Neurocritical Care Society, a multidisciplinary non-profit organisation with thousands of members around the world, is poised to take advantage of the new neurotechnologies..The society undertakes research through the Neurocritical Care Research Network. ${ }^{5}$ The fresh impetus for such research initiatives is the desire of clinical scientists to enhance our understanding of complex disease states to improve patient outcomes and maintain brain health. The main goal of the society was to foster collaborative multidisciplinary clinical research and to advance critical care research methods such as using specific integrated chips for monitoring patients with traumatic brain injury. By monitoring the electrocorticography and neurochemical signals of the injured human brain tissue, it might be possible to detect spreading depolarisations, which are associated with poor outcomes in patients with traumatic brain injury. The "behind the ear" wireless microplatform device also enables monitoring of mobile patients with traumatic brain injury for secondary brain injury impact. ${ }^{6}$ Recent multidisciplinary collaborative clinical research indicates that a better outcome for patients in the completely locked-in state (severe disability) or with severe stroke may be feasible using brain-computer interface training to improve motor rehabilitation. ${ }^{7}$

\section{Need for standardisation}

Further challenges are raised by the varying directions of brain research projects around the world. They have different funding mechanisms, project management structures, and approaches to ethical issues. It is imposible to achieve an understanding of the mysteries of the brain in one project alone-integrated collective intellectual and technological support are needed from different resources. Enhanced standardisation of those elements that enable scientists to compare data and contribute to building a common knowledge base of the brain is urgently needed.

What should be standardised to construct a framework that will bring together 
the results of large scale brain research initiatives from different countries? Examples might include agreement on common core acquisition methods and sharing plans for human brain imaging and data, or common standards for meta data and analysis tools for single cell typing studies. Also included could be approaches to disseminate new neurotechnologies and training programmes to optimise their application to brain projects. Human genetics has already benefited from global team science. An excellent model is ENIGMA (Enhancing Neuroimaging Genetics through Meta-Analysis), a worldwide network of researchers who pool brain imaging and genetic data from over 200 institutions aiming to investigate various aspects of the brain. ${ }^{8}$

Alzheimer's disease is one disease where such networks could advance understanding. Amyloid deposition, intracellular tau aggregates, vascular compromise, and immune/inflammatory alteration are strongly implicated in the pathogenesis of neurodegeneration in Alzheimer's disease. Sleep disturbance, changes in the brain's glymphatic flow, and even effects of microbiota on brain functioning provide other contributory factors. Yet despite this new knowledge, effective therapies have been elusive. Tools from the global brain initiatives that enable investigators to interrogate the complex circuits affected in Alzheimer's disease should enable the science to move from associative molecular-structural relationships to treatments that intervene to preserve circuit function.

Common worldwide data may provide insight into additional therapeutic targets, which mainly focus on transforming basic research achievements into clinical prevention and treatment. Further research into degenerative medicine, vascular biology, public health sciences, and clinical trial implementation and organisation is also important. ${ }^{9}$

As with earlier projects in genomics, astronomy, and physics, the enthusiasm of brain initiatives around the world call for strengthening international collaboration. The aim is to reduce the current and future brain related disease burden through multidisciplinary research and capacity building, promoting the development of effective prevention and intervention for neurological disorders. Box 1 summarises the challenges and opportunities regarding brain research, especially for global collaboration.

\section{Models of collaboration}

What models for international collaboration might neuroscientists emulate to achieve productive research worldwide? Consultation on all potential elements would requires involvement of a wide range of stakeholders from academia, industry, and government.

To tackle this challenging task, we suggest identifying the core areas of research priorities, expanding scientific opportunities, and disseminating discoveries for the benefit of humanity. The most notable example of such collaboration in our area of medicine is the BRAIN initiative, a partnership between the National Institutes of Health, the National Science Foundation, the Defence Advanced Research Projects Agency, private foundations, and researchers. ${ }^{10}$ We have a limited understanding of brain function and the workings of neural networks. The development and application of innovative technologies that explore brain circuits over the spatial scales that range from moleculular interactions at the synapse to electron microscopic level connections, and then to mesoscale imaging of structural and functional neuroimaging will result in a dynamic picture of brain function.

As an example, gait deficits contribute significantly to functional disability after stroke. Recent technological advances in stroke gait rehabilitation have made it possible for robotic devices to provide safe, intensive training through accurate repetitive motion. ${ }^{11}$ There is evidence that electrical stimulation of the brain, as a means to further engage post-stroke neuroplasticity and enhance functional recovery, may promote recovery and improvement in symptoms. Various neuromodulation techniques are under investigation for stroke patients, including transcranial direct current stimulation,

Box 1: Challenges and opportunities of future global collaboration on brain research

Emerging challenges call for global collaboration for brain health research

- Limited resources and knowledge about the mechanisms of brain function and dysfunction

- Few integrated projects provide insights into the priority and benefit of human brain research worldwide

- International collaborative projects required to treat devasating neurological and mental health disorders that are major social and economic burdens on society

Opportunities of global collaboration on brain research

- Organising the multidisciplinary high level basic or clinical research worldwide to take advantage of core research direction from every resource

- Distributing novel technologies and sharing the generated data worldwide

- The International Brain Research Organization and the International Brain Inititiave could provide support for relevant issues across the world, such as policy and ethics 
technology for informatics platforms are critical to support modelling and theory development. The Human Brain Project's platforms give scientists a single point of access to neuroscientific method, multiomic clinical data, and analysis tools from around the world. ${ }^{15}$ Thanks to the international collaborative projects, the field of functional neuroimaging has advanced substantially, showing the value of big data science. ${ }^{16}$ On the clinical side we have seen the value of harmonisation of variables among relevant studies to promote greater comparability across collaborating research projects. ${ }^{17}$

Machine learning and artificial intelligence techniques based on big data are increasingly being used in both understanding and diagnosis of neurological disorders and offer a new model for personalised management. Machine learning techniques could be used to delineate the categories and predict the patients' outcomes with various conditions. For example, artificial intelligence has been successfully used on pattern recognition of electroencephalogram or neuroimaging abnormalities for diagnostic purposes in patients with epilepsy. ${ }^{18}$

However, these efforts are only the beginning. A synergistic international effort could provide greater global impact and better use of precious research funding, including government, industry, non-governmental organisations, and individual contributions. It requires rewarding the groups or team for the collective effort rather than a few lead investigators. In the international brain project, expertise is unlikely to lie in a single country. The removal of national barriers for funding team science seems a desirable goal but is difficult politically and even more difficult when it entails intellectual property claims. The Human Brain Project exemplified team science funding within the European Union; the US BRAIN initiative makes funding available to any researcher from any country, as long as the proposed project is deemed worthy by the study sections that review it.

Importantly, new tools to map, monitor, and modulate brain circuits in humans hold great promise not only for the treatment of neurological disorders but also the ethical challenge to understand how these tools should be used. The answers may depend on cultural beliefs, but the processes for establishing ethical guidelines should be global and transparent. Many countries have incorporated ethical issues into the design of their brain programmes. ${ }^{19}$

In conclusion, the collective success of bridging these projects into a global collaboration that aims to understand the scientific basis of brain structure and function could have a key role in this era of academic development. In addition, it would be of benefit for science as a whole, open up a new strategic direction and promotion for brain disease prevention, create new industries, and ultimately achieve a better life for individuals and the population.

Contributors and sources: All the authors proposed the idea of this manuscript. $L L$ drafted the first version and all the authors critically reviewed and revised the manuscript. LL is director of the neurocritical care unit at Beijing Tiantan Hospital and her focus is on stroke care and clinical research. VF's research is focused on global epidemiology and prevention of neurological disorders, with a particular focus on stroke. RLS is executive director of the McKnight Brain Institute and director of the Miami Clinical and Translational Science Institute and immediate past-president of the American Academy of Neurology. WJK is co-director of the NIH BRAIN Initiative and previously worked in stroke and neurointensive care services.

Competing interests: We have read and understood the BMJ policy on declarations of interest and declare that the study was supported by grants from the Ministry of Science and Technology of China (2016YFC1307301) and National Natural Science Foundation of China (81820108012).

Provenance and peer review: Commissioned; externally peer reviewed.

This article is part of a series launched at the Chinese Stroke Association annual conference on 10 October 2020, Beijing, China. Open access fees were funded by the National Science and Technology Major Project. The BM/ peer reviewed, edited, and made the decision to publish these articles.

Liping Liu, professor ${ }^{1,2}$

Valery Feigin, professor $^{3}$

Ralph L Sacco, professor ${ }^{4}$

Walter J Koroshetz, director ${ }^{5}$

${ }^{1}$ Department of Neurology, Beijing Tiantan Hospital, Capital Medical University, Beijing, China

${ }^{2}$ China National Clinical Research Center for Neurological Diseases, Beijing, China

${ }^{3}$ National Institute for Stroke and Applied Neurosciences, School of Clinical Sciences, Auckland University of Technology, Auckland, New Zealand

${ }^{4}$ Department of Neurology, Miller School of Medicine, University of Miami, Miami, FL, USA

${ }^{5}$ National Institute of Neurological Disorders and Stroke, NIH, Bethesda, MD, USA

Correspondence to: Walter J Koroshetz koroshetzw@ninds.nih.gov

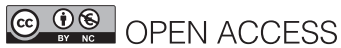

This is an Open Access article distributed in accordance with the Creative Commons Attribution
Non Commercial (CC BY-NC 4.0) license, which permits others to distribute, remix, adapt, build upon this work non-commercially, and license their derivative works on different terms, provided the original work is properly cited and the use is non-commercial. See: http://creativecommons.org/ licenses/by-nc/4.0/.

\section{Check for updates}

1 Stein JL, Medland SE, Vasquez AA, et al, Alzheimer's Disease Neuroimaging Initiative, EPIGEN Consortium, IMAGEN Consortium, Saguenay Youth Study Group, Cohorts for Heart and Aging Research in Genomic Epidemiology Consortium, Enhancing Neuro Imaging Genetics through Meta-Analysis Consortium. Identification of common variants associated with human hippocampal and intracranial volumes. Nat Genet 2012;44:552-61. doi:10.1038/ng.2250

2 Adams A, Albin S, Amunts K, et al, International Brain Initiative. Electronic address: j.g.bjaalie@medisin.uio. no, International Brain Initiative. International Brain Initiative. An innovative framework for coordinated global brain research efforts. Neuron 2020;105:212 6. doi:10.1016/j.neuron.2020.01.002

3 Huang ZI, Luo L. NEUROSCIENCE. It takes the world to understand the brain. Science 2015;350:42-4. doi:10.1126/science.aad4120

4 Yuste R, Bargmann C. Toward a global BRAIN initiative. Cell 2017;168:956-9. doi:10.1016/j. cell.2017.02.023

5 Suarez Jl, Geocadin R, Hall C, et al. The neurocritical care research network: NCRN. Neurocrit Care 2012;16:29-34. doi:10.1007/s12028-0119612-x

6 Pagkalos I, Rogers ML, Boutelle MG, Drakakis EM. A high-performance application specific integrated circuit for electrical and neurochemical traumatic brain injury monitoring Chemphyschem 2018;19:1215-25. doi:10.1002/ cphc.201701119

7 Chaudhary U, Birbaumer N, Ramos-Murguialday A. Brain-computer interfaces in the completely locked-in state and chronic stroke. Prog Brain Res 2016;228:131-61. doi:10.1016/ bs.pbr.2016.04.019

8 Thompson PM, Hibar DP, Stein JL. Genetics of the connectome and the ENIGMA project. In: Kennedy $\mathrm{H}$, Van Essen DC, Christen Y, eds. Micro-, meso- and macro-connectomics of the brain. Springer; 2016.

9 Elliott LT, Sharp K, Alfaro-Almagro F, et al. Genome-wide association studies of brain imaging phenotypes in UK Biobank. Nature 2018;562:210-6. doi:10.1038/s41586-018-0571-7

10 Insel TR, Landis SC, Collins FS, The NIH BRAIN Initiative. Research priorities. Science 2013;340:687-8. doi:10.1126/ science. 1239276

11 Lee HJ, Lee SH, Seo K, et al. Training for walking efficiency with wearable hip-assist robot in patients with stroke: A pilot randomized controlled trial. Stroke 2019;50:3545-52. doi:10.1161/ STROKEAHA.119.025950

12 Elias GJB, Namasivayam AA, Lozano AM. Deep brain stimulation for stroke: Current uses and future directions. Brain Stimul 2018;11:3-28. doi:10.1016/j.brs.2017.10.005

13 Martinez-Ramirez D, Jimenez-Shahed J, Leckman JF et al. Efficacy and safety of deep brain stimulation in Tourette syndrome: the International Tourette Syndrome Deep Brain Stimulation Public Database and Registry. JAMA Neurol 2018;75:353-9. doi:10.1001/jamaneurol.2017.4317

14 Zivadinov $\mathrm{R}$. Why is cognitive impairment present in multiple sclerosis? Insights from functional 


\section{BRAIN HEALTH}

MRI. Radiology 2018;288:552-3. doi:10.1148/ radiol.2018180719

15 Poo MM, Sander L, Fivaz M, Hayashi Y. Asian promise: the state and future of collaborations in neuroscience. Nat Rev Neurosci 2008;9:881-4. doi:10.1038/nrn2512

16 Li X, Guo N, Li Q. Functional neuroimaging in the new era of big data. Genomics Proteomics Bioinformatics 2019;17:393-401. doi:10.1016/j. gpb.2018.11.005
17 Meeuws S, Yue JK, Huijben JA, et al. Common data elements-critical assessment of harmonization between current multicenter traumatic brain

injury studies. J Neurotrauma 2020;37:1283-90. doi:10.1089/neu.2019.6867

18 Pitkänen A, Löscher W, Vezzani A, et al. Advances in the development of biomarkers for epilepsy. Lancet Neurol 2016;15:843-56. doi:10.1016/S14744422(16)00112-5
19 Hyun I, Scharf-Deering IC, Lunshof JE. Ethical issues related to brain organoid research. Brain Res 2020;1732:146653. doi:10.1016/j. brainres.2020.146653

Cite this as: $B M / 2020 ; 371: \mathrm{m} 3753$

http://dx.doi.org/10.1136/bmj.m3753 